\title{
Détection optique de la résonance magnétique de centres profonds dans les semiconducteurs $(*)$
}

\author{
R. T. Cox $\left({ }^{* *}\right)$ \\ Département de Recherche Fondamentale, \\ Centre d'Etudes Nucléaires de Grenoble, 85X, 38041 Grenoble Cedex, France
}

\begin{abstract}
Résumé. - Lors d'un événement de recombinaison radiative au niveau d'un centre profond, l'émission optique correspondante est constituée très souvent d'une bande large sans aucune structure résolue. Il peut alors être difficile (voire impossible) d'identifier, par la spectroscopie optique seule, les centres responsables de ces bandes. C'est notamment le cas dans les phénomènes de recombinaison donneur-accepteur lorsque le donneur ou l'accepteur est un centre profond. Depuis 1975, la résonance magnétique détectée optiquement (RMDO) s'est avérée très utile dans ce type de problème. Nous discuterons de l'emploi de la RMDO pour l'identification des centres de luminescence et pour l'étude de leurs propriétés physiques dans les semiconducteurs, principalement des composés II-VI.
\end{abstract}

Abstract. - When radiative recombination takes place at a deep centre, the corresponding optical emission
consists very often of a broad band, without any resolved structure. It can then be very difficult (or impossible)
to identify the centres responsible for the emission. An important example is the case of donor-acceptor recombina-
tion when either the donor or the acceptor is a deep centre. Since 1975, optically detected magnetic resonance
(ODMR) has proved to be very useful in this type of problem. A review is given of the use of ODMR for identi-
fying luminescence centres and for studying their physical properties in semiconductors, mainly II-VI compounds.

La spectroscopie de luminescence constitue souvent une technique de choix pour étudier les impuretés et autres défauts présents dans un cristal semiconducteur : citons les très nombreuses études de recombinaison radiative d'excitons piégés ou de luminescence donneur-accepteur (luminescence D-A), par exemple celles qui ont permis récemment d'identifier les centres responsables de la conductibilité de type $\mathrm{p}$ de $\mathrm{ZnTe}$ $[1,2]$. Cependant, cette technique ne montre son potentiel maximum que lorsqu'il est possible d'observer des raies d'émission très fines, les raies zérophonon. Or, dans le cas d'une recombinaison radiative au niveau d'un centre profond ou d'une transition optique interne d'un centre profond, on n'observe souvent qu'une bande d'émission très large, conséquence des fortes interactions entre un électron ou un trou très localisé et les noyaux environnants. Il devient alors impossible de déterminer avec précision les énergies des niveaux électroniques, d'observer l'effet Zeeman, etc. En conséquence, l'interprétation de

(*) Conférence présentée au Congrès de la Société Française de Physique (Toulouse).

$\left({ }^{* *}\right)$ Chercheur C.N.R.S. bandes optiques de ce type est très difficile et peut donner lieu à de longues controverses.

Depuis 1975, la technique de détection optique de la résonance paramagnétique électronique (RPE), ou plus brièvement résonance magnétique détectée optiquement (RMDO), s'est avérée extrêmement utile dans un certain nombre de cas de ce type. En permettant d'observer la RPE de centres dans leur état excité luminescent, elle les identifie avec précision tout en fournissant des renseignements intéressants sur leurs propriétés physiques.

Nous passerons en revue les résultats de RMDO portant sur les centres profonds. Les résultats publiés à ce jour (juin 1979) concernent presque uniquement des centres accepteurs et donneurs responsables de luminescence D-A. Cependant, la méthode devrait également être très utile pour interpréter des transitions internes de centres profonds, comme elle l'a été précédemment en physique des cristaux ioniques (voir par exemple les travaux sur l'état triplet excité de la lacune d'oxygène dans $\mathrm{CaO}$ [3-5]). D'autre part, la RMDO est utilisée pour étudier les transitions optiques en bord de bande, c'est-à-dire des transitions de centres peu profonds $[6,7]$. En fait, la première application de cette technique en physique des semicon- 
ducteurs a été la mesure du facteur $y$ d'électrons de conduction $[8,9]$.

1. Principe d'une expérience de RMDO. - Dans une expérience de RMDO, les transitions provoquées par une source hyperfréquence entre les sous-niveaux Zeeman d'un état excité luminescent sont détectées par l'intermédiaire des changements résultant d'intensité ou de polarisation de la luminescence. Ces changements ont pour origine le fait que la probabilité d'émission optique peut être différente pour chaque sous-niveau Zeeman. La figure 1 donne le principe d'une expérience de ce type.

Comme dans un spectromètre de RPE classique, l'échantillon est placé dans une cavité hyperfréquence liée à une source (par exemple un klystron travaillant à $9 \mathrm{GHz}$ ). Cependant, le dispositif hyperfréquence peut être nettement plus simple qu'en RPE classique puisqu'un pont hyperfréquence avec détecteur à diode pour mesurer l'absorption hyperfréquence n'est pas nécessaire (à moins que l'on ne veuille faire simultanément RPE classique et RMDO). La cavité est placée dans un champ magnétique $H$ fourni par un électroaimant ou une bobine supraconductrice.

La luminescence de l'échantillon semiconducteur est généralement excitée avec un laser continu à gaz; son intensité $I$ est mesurée à l'aide d'un photomultiplicateur. Les variations de $I$ induites par la source sont enregistrées en faisant varier $H$ : un spectre type (que nous commenterons plus tard) est représenté à droite de la figure 1. En réalité, le système de détection est légèrement plus compliqué que ne le montre la figure. Les signaux de RMDO ayant fréquemment un faible rapport signal/bruit et étant souvent transitoires, on insère un modulateur hyperfréquence

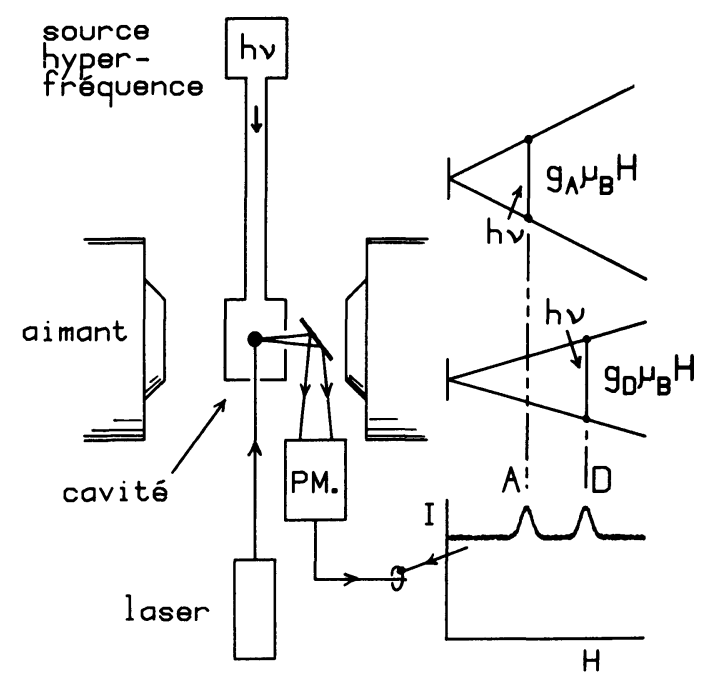

Fig. 1. - Appareillage d'une expérience de résonance magnétique détectée optiquement. A droite : spectre type obtenu dans le cas d'une luminescence D-A.

[Apparatus for optical detection of magnetic resonance (ODMR). A typical spectrum obtained in the case of a D-A luminescence is shown at the right.] (diode PIN) après la source hyperfréquence afin de moduler (en créneaux à $f=10^{2}-10^{5} \mathrm{~Hz}$ ) la puissance incidente sur la cavité. Le signal électrique donné par le PM est démodulé à l'aide d'une détection synchrone et on obtient directement la variation $\Delta I$ provoquée par l'onde hyperfréquence, c'est-à-dire le spectre de RMDO.

Si l'on désire analyser l'effet de l'onde hyperfréquence sur le taux de polarisation circulaire de l'émission, on utilise le montage optique de la figure 1, qui permet d'observer la luminescence émise parallèle à $H$. Dans les cas décrits dans cet article, l'effet sur la polarisation circulaire est très faible et la direction d'observation peut être quelconque.

Enfin, pour maximiser les différences de population entre les sous-états Zeeman de l'état excité, l'échantillon doit être refroidi, généralement à la température de l'hélium liquide.

Signalons qu'il est souvent possible d'observer la RPE de centres créés par photoexcitation en utilisant un spectromètre de RPE classique (en irradiant l'échantillon dans la cavité). En fait, l'interprétation de la plupart des spectres RMDO décrits ci-après est basée sur les résultats d'expériences de RPE classiques faites sur les mêmes types de cristaux. Précisons donc l'intérêt de la RMDO par rapport à la RPE classique.

Premièrement, les détecteurs de lumière étant beaucoup plus sensibles que les détecteurs hyperfréquence, la RMDO peut être plus sensible que la RPE classique pour observer des états excités métastables. Deuxièmement, sauf complication (voir plus loin), en RMDO on ne détecte que les centres responsables de la luminescence. Dans la mesure où l'on peut identinier le spectre de RMDO, on pourra ensuite interpréter le phénomène de luminescence.

Afin de corréler une bande de luminescence et un spectre de RMDO, on insère un monochromateur entre l'échantillon et le détecteur PM sur la figure 1. Ainsi, on peut déterminer la variation du signal de RMDO en fonction de la longueur d'onde d'émission. Ceci constitue une technique puissante pour résoudre des bandes d'émission qui se recouvrent. Signalons néanmoins une source possible de complications. Par des processus complexes de cross-relaxation ou de transfert d'excitation optique, le fait d'induire des transitions de RPE d'un type de centre peut quelquefois modifier la luminescence d'un autre type de centre. Ceci a été observé par Boccara et Fournier [10] dans le cas de cristaux de $\mathrm{ZnS}$ dopés au manganèse : le spectre de RMDO obtenu sur la luminescence selfactivée (luminescence $\mathrm{D}$-A) de l'échantillon est identique au spectre de RPE de l'ion $\mathrm{Mn}^{2+}$ dans son état fondamental.

2. RMDO d'une paire donneur-accepteur profond. - Nous rappelons d'abord les propriétés essentielles $\mathrm{du}$ phénomène de luminescence $\mathrm{D}$-A [11]. Cette luminescence peut être obtenue par exemple en irradiant l'échantillon semiconducteur avec des photons 
d'énergie suffisante pour créer des électrons et des trous libres $\left(h v>E_{\text {gap }}\right)$. Si l'échantillon contient des donneurs ionisés $\mathrm{D}^{+}$compensés par des accepteurs ionisés $\mathrm{A}^{-}$, ceux-ci peuvent capturer respectivement les électrons et les trous et on obtient ainsi une concentration métastable de donneurs neutres $D^{0}$ et d'accepteurs neutres $A^{0}$. L'électron d'un donneur peut se recombiner alors avec le trou situé sur un accepteur qui se trouve dans son voisinage, du fait du recouvrement entre les deux fonctions d'onde, cela après un délai qui augmente exponentiellement avec la distance $r_{\text {DA }}$. Chaque transition de ce type correspond donc au retour d'une paire donneur-accepteur $\mathrm{D}^{0}, \mathrm{~A}^{0}$ vers son état fondamental $\mathrm{D}^{+}, \mathrm{A}^{-}$, avec émission d'un photon d'énergie :

$$
h v=E_{\mathrm{gap}}-E_{\mathrm{D}}-E_{\mathrm{A}}+E\left(r_{\mathrm{DA}}\right)-E_{\mathrm{vib}}
$$

où $E_{\mathrm{D}}, E_{\mathrm{A}}$ sont les profondeurs thermiques des niveaux et $E\left(r_{\mathrm{DA}}\right)$ est une correction, fonction de $r_{\mathrm{DA}}$, due aux interactions donneur-accepteur. Le terme $E_{\text {vib }}$ représente la différence d'énergie entre l'état vibrationnel terminal de la transition optique et l'état vibrationnel fondamental correspondant à la configuration $\mathrm{D}^{+}$, $\mathrm{A}^{-}$. Pour une raie zéro-phonon, $E_{\mathrm{vib}}=0$. Cependant, dans les cas qui nous intéressent ici, où l'un au moins des deux centres $\mathrm{D}^{0}$ ou $\mathrm{A}^{0}$ est un centre profond, on n'observe pas de raie zéro-phonon puisque la position moyenne de certains noyaux doit être très différente entre l'état $\mathrm{D}^{0}, \mathrm{~A}^{0}$ et l'état $\mathrm{D}^{+}, \mathrm{A}^{-}$; on observe alors une bande d'émission optique très large ( $\Delta E \simeq 0,4 \mathrm{eV}$ typiquement) située à énergie plus basse, qui correspond à des transitions optiques qui sont accompagnées de l'émission d'un grand nombre de phonons.

Etudions maintenant l'effet du champ magnétique $H$ dans le cas où $\mathrm{D}^{+}$et $\mathrm{A}^{-}$sont des défauts à couches électroniques complètes. Le donneur neutre $\mathrm{D}^{0}$ est donc un donneur simple possédant un électron célibataire qui lui confère un spin $S_{\mathrm{D}}=1 / 2$. De même, le spin total de l'accepteur neutre $\mathrm{A}^{0}$ est $S_{\mathrm{A}}=1 / 2$, puisqu'on a un trou dans des couches complètes.

Dans un cristal cubique, l'accepteur $\mathrm{A}^{0}$ peut également posséder un moment orbital $L=1$ (lié à la dégénérescence de la bande de valence); le couplage spin-orbite donne alors les états spin-orbite $J=1 / 2$ et $J=3 / 2$ bien connus. Cependant, il est rarement possible d'observer la RPE d'un état $L=1$, car les contraintes présentes dans tout échantillon séparent les trois sous-états de ce triplet orbital produisant des décompositions aléatoires qui sont comparables ou supérieures aux décompositions créées par le champ $H$. C'est l'une des raisons pour lesquelles on n'a pas encore observé la RMDO d'accepteurs peu profonds. Cependant, dans un accepteur profond, le trou étant très localisé peut interagir fortement avec des distorsions trigonales ou tétragona'es de son environnement. Il s'ensuit une distorsion spontanée (effet Jahn-Teller [12] ou pseudo-Jahn-Teller [13]) qui décompose l'état $L=1$, la levée de dégénérescence étant dans ce cas de l'ordre de $1 \mathrm{eV}$. Ceci supprime le moment orbital, ne laissant que la dégénérescence de spin : l'accepteur profond se trouve donc dans un état avec $L=0, S=1 / 2$ dont les propriétés de RPE sont similaires à celles d'un donneur (pour la plupart des donneurs dans les semiconducteurs, l'état fondamental ne possède que la dégénérescence de spin).

Dans le champ $H$, les spins $S_{\mathrm{D}}$ et $S_{\mathrm{A}}$ s'orientent parallèlement ou antiparallèlement à $H(\uparrow$ ou $\downarrow)$. A droite de la figure 1 , nous indiquons les dédoublements Zeeman $g_{\mathrm{D}} \mu_{\mathrm{B}} H$ et $g_{\mathrm{A}} \mu_{\mathrm{B}} H$ des deux doublets de spin. En général, l'effet du couplage spin-orbite est tel que $g_{\mathrm{D}}<2$ et $g_{\mathrm{A}}>2$; ceci permet de bien distinguer les deux types de centre. En additionnant les énergies Zeeman respectives, on obtient l'énergie totale des quatre configurations possibles des deux spins, cf. la partie gauche de la figure 2. L'énergie de chacun des quatre niveaux magnétiques de la figure 2 est simplement

$$
E=g_{\mathrm{D}} \mu_{\mathrm{B}} M_{\mathrm{D}} H+g_{\mathrm{A}} \mu_{\mathrm{B}} M_{\mathrm{A}} H
$$

où $M_{\mathrm{D}}, M_{\mathrm{A}}= \pm 1 / 2$. Pour l'instant, nous négligeons toute interaction entre les deux spins $(J=0$ sur la figure 2).

Or, la luminescence D-A est une émission de type dipolaire électrique. Au cours d'une telle transition, si on néglige les effets (faibles dans les cas étudiés ici) du couplage spin-orbite, l'état de spin ne peut pas

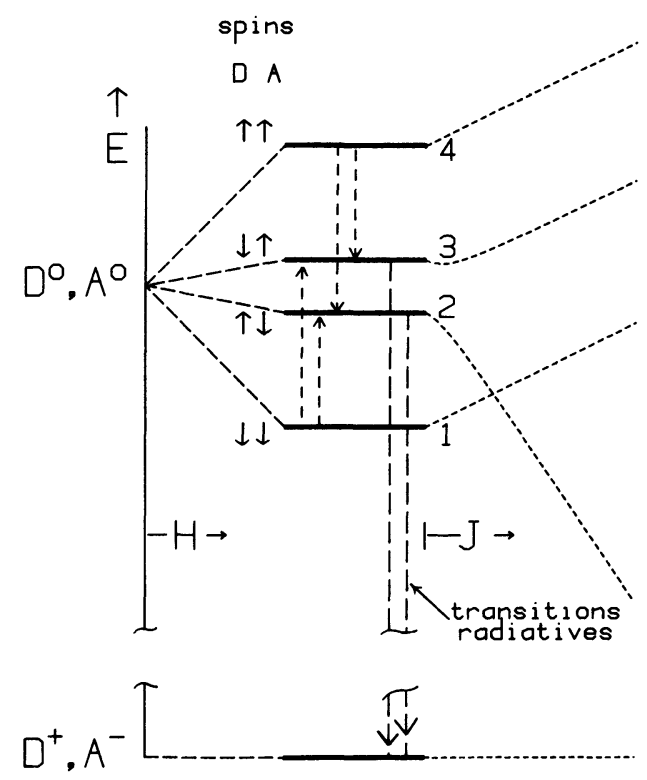

Fig. 2. - Niveaux d'énergie d'une paire D-A dans son état fondamental $\mathrm{D}^{+}, \mathrm{A}^{-}$et dans son état excité $\mathrm{D}^{0}, \mathrm{~A}^{0}$. A gauche : niveaux d'énergie des quatre configurations de spin en présence d'un champ magnétique $H$; transitions hyperfréquences; transitions de luminescence. A droite : effet d'une interaction d'échange $J$ entre le donneur et l'accepteur.

[Energy levels of a D-A pair in the ground state $\mathrm{D}^{+}, \mathrm{A}^{-}$and in the excited state $D^{0}, A^{0}$. At left : energies of the four spin configurations in a magnetic field $H$; microwave transitions ; luminescence transitions. At right : effect of donor-acceptor exchange interaction $J$.] 
être modifié (règle de sélection $\Delta M_{\mathrm{S}}($ total $)=0$ ). Puisque tous les spins sont appariés dans l'état final à couches électroniques complètes $\mathrm{D}^{+}, \mathrm{A}^{-}$, seules les paires $\mathrm{D}^{0}, \mathrm{~A}^{0}$ qui ont leurs spins antiparallèles $(\downarrow \uparrow$ ou $\uparrow \downarrow)$ peuvent se désexciter radiativement. Les paires $\mathrm{D}^{0}, \mathrm{~A}^{0}$ avec leurs spins parallèles se trouvent dans des états non radiatifs, voir la figure 2 .

Si maintenant on applique une onde hyperfréquence de fréquence $v$ avec, par exemple, $h v=g_{\mathrm{D}} \mu_{\mathrm{B}} H$ (comme on l'a indiqué à droite sur la figure 1), on va pouvoir renverser le spin d'un donneur d'une paire non radiative $\uparrow \uparrow$ ou $\downarrow \downarrow$, convertissant celle-ci en paire radiative. Cependant, la probabilité de transition hyperfréquence étant la même dans les deux sens, la même source hyperfréquence transformera également des paires radiatives en paires non radiatives. Pour qu'il existe globalement un effet sur l'intensité de la luminescence, il faut que la population des paires avec spins parallèles soit différente de celles des paires avec spins antiparallèles. Cette différence existe effectivement, ce qui permet de détecter la RPE du donneur sous la forme d'un changement d'intensité de la luminescence D-A. De même, avec $h v=g_{\mathrm{A}} \mu_{\mathrm{B}} H$, on observe la RPE de l'accepteur. Dans la pratique, on constate une augmentation de l'intensité lumineuse, ce qui conduit au spectre de RMDO type de la figure 1 .

Nous allons maintenant décrire les principaux résultats portant sur la luminescence de centres profonds.

3. ZnS : luminescence self-activée. - La luminescence bleue dite self-activée de $\mathrm{ZnS}$ possède un grand intérêt historique dans les études des bandes d'émission des semiconducteurs II-VI. De plus, elle constitue la première luminescence de ce type à avoir été étudiée par RMDO $[14,15,16]$.

De nombreux phénomènes de luminescence nécessitent la présence de deux types d'impuretés, les activateurs et les co-activateurs : ceux-ci se sont révélés être respectivement des accepteurs et des donneurs, ce qui a conduit Prener et Williams [17] à inventer le modèle de luminescence donneur-accepteur. Par contre, d'autres bandes de luminescence, baptisées self-activées, ne nécessitent qu'une impureté co-activatrice (un donneur). Des études de RPE classique [18-21] sous excitation lumineuse ont montré la présence d'un centre accepteur intrinsèque dans des échantillons de $\mathrm{ZnS}$ présentant une luminescence self-activée. Ce centre, la lacune de zinc une fois ionisée $\mathrm{V}_{\mathrm{Zn}}^{-}$, est associé presque toujours avec un donneur ionisé $\mathrm{D}^{+}$en site proche voisin. On a proposé que cet accepteur complexe $A^{0}=\left(V_{\mathrm{Zn}} \mathrm{D}\right)^{0}=\left(\mathrm{V}_{\mathrm{Zn}}^{-} \mathrm{D}^{+}\right)$ soit un activateur de luminescence.

Avant 1975, la luminescence self-activée était interprétée de deux façons différentes (a) comme une luminescence donneur-accepteur

$$
\mathrm{D}^{0},\left(\mathrm{~V}_{\mathrm{Zn}} \mathrm{D}\right)^{0} \rightarrow \mathrm{D}^{+},\left(\mathrm{V}_{\mathrm{Zn}} \mathrm{D}\right)^{-}
$$

et (b) comme une transition interne du complexe accepteur ionisé $\left(\mathrm{V}_{\mathrm{Zn}} \mathrm{D}\right)^{-*} \rightarrow\left(\mathrm{V}_{\mathrm{Zn}} \mathrm{D}\right)^{-}$[22]. Les résul- tats obtenus par James et coll. $[14,15]$ et Nicholls et coll. [16] ont montré que le modèle (a) est juste.

La figure 3 montre un spectre de RMDO d'un échantillon de $\mathrm{ZnS}$ (Eagle-Picher) détecté par l'intermédiaire de la luminescence self-activée. Le spectre est très similaire à ceux publiés dans les références [14-16] et correspond au spectre type indiqué sur la figure 1.

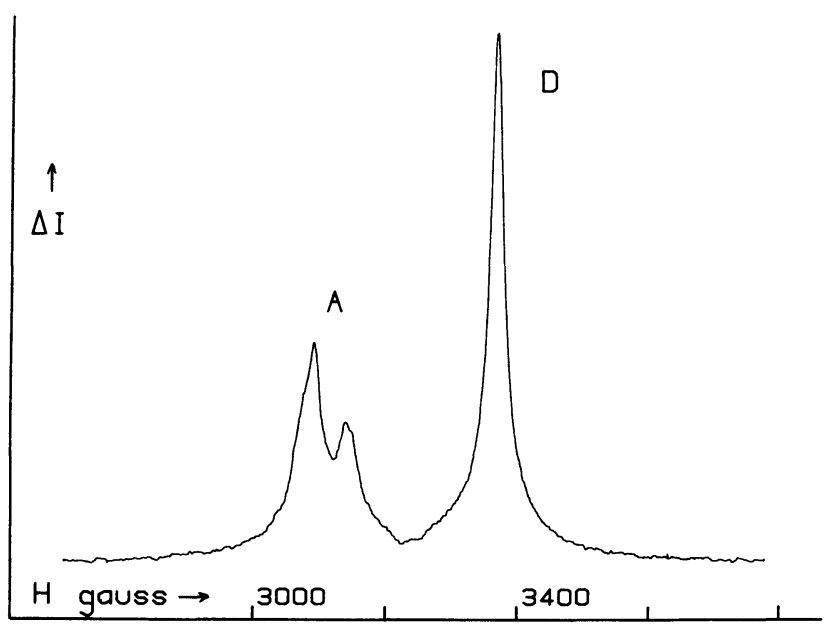

Fig. 3. - Spectre de RPE détectée optiquement par l'intermédiaire de la luminescence self-activée d'un échantillon de $\mathrm{ZnS}$; fréquence de la source hyperfréquence $=9,0 \mathrm{GHz} ; T=2 \mathrm{~K}$. La raie $\mathrm{D}$ correspond à un donneur peu profond, le groupe de raies $\mathrm{A}$ à la lacune de zinc.

[EPR spectrum detected optically via the self-activated luminescence of a $\mathrm{ZnS}$ sample. Microwa ve frequency $9 \mathrm{GHz} ; T=2 \mathrm{~K}$. The line D corresponds to a shallow donor, line $\mathrm{A}$ to the zinc vacancy.]

On y observe une raie simple $\mathrm{D}$ à $g=1,89$ et une raie structurée $\mathrm{A}$ dans la région $g=2$ à 2,05. Par comparaison avec les résultats de RPE classique obtenus par Müller et Schneider [24] sur ZnS photoexcité, les auteurs cités [14-16] ont attribué la raie D à un donneur peu profond. Remarquons que dans $\mathrm{ZnS}$ les différentes espèces de donneur peu profond, ainsi que les électrons de conduction, ont leur facteur $g$ très voisin. Malheureusement, la largeur importante de la raie de RMDO, de l'ordre de $40 \mathrm{G}$ sur la figure 3 (comparée à $1 \mathrm{G}$ environ en RPE classique), empêche de faire les mesures très précises qui pourraient permettre d'identifier la nature chimique du donneur. Il en sera de même pour les donneurs peu profonds dans $\mathrm{ZnO}$ et $\mathrm{ZnSe}$.

La comparaison avec les spectres de RPE classique de lacunes de zinc [18-21] montre que la raie $A$ correspond à un centre $V_{\mathbf{Z}_{n}}^{-}$, faisant probablement partie

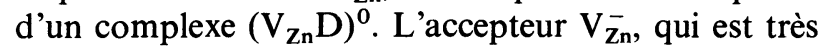
profond $\left(E_{\mathrm{A}}\right.$ de l'ordre de $\left.1 \mathrm{eV}\right)$, subit une distorsion pseudo-Jahn-Teller qui localise le trou dans une orbitale $3 p$ d'un soufre du tétraèdre contenant la lacune. Il existe donc quatre possibilités de localisation différentes qui donnent quatre raies de RPE puisque le facteur $g_{\mathrm{A}}$ dépend de l'angle entre $H$ et l'axe de 
l'orbitale $3 \mathrm{p}$. Les études de RPE classique mettent en évidence des décompositions supplémentaires dues à l'effet perturbateur du donneur $\mathrm{D}^{+}$voisin. Cette structure complexe de raies n'est pas résolue en RMDO : la raie A de la figure 3 constitue son enveloppe.

L'observation de la RPE du donneur et de l'accepteur par l'intermédiaire de la luminescence self-activée a permis d'attribuer cette luminescence à une recombinaison entre un donneur peu profond et une lacune de zinc. Signalons que cette identification repose en partie sur l'attribution de la largeur importante des raies de RMDO aux interactions d'échange donneuraccepteur qui (comme nous le verrons plus loin) possèdent tout un spectre de valeurs possibles, permettant ainsi de lever l'ambiguïté entre un électron de conduction et un donneur neutre.

4. ZnSe : luminescence self-activée. - Ce système est très similaire à celui décrit précédemment. La luminescence en question est constituée d'une bande rouge centrée vers $2,0 \mathrm{eV}$ dont les caractéristiques précises varient avec la nature exacte du donneur et de l'accepteur. Par RMDO, on détecte un spectre similaire à celui de $\mathrm{ZnS}$, constitué par la raie d'un donneur peu profond $\left(g_{\mathrm{D}}=1,13\right)$ et par les raies de la lacune de zinc $\mathrm{V}_{\mathrm{Zn}}^{-}$(dans la région $\left.g=1,96-2,25\right) \quad[25,26]$. La résolution des raies de l'accepteur étant meilleure que dans le cas de $\mathrm{ZnS}$, il est possible d'après les auteurs cités de distinguer la RMDO de lacunes isolées $\mathrm{V}_{\mathbf{Z n}}^{-}$(identifiées précédemment par RPE classique par Watkins [27]) de la RMDO de complexes $\mathrm{V}_{\mathrm{Zn}}^{-} \mathrm{D}^{+}[20]$. Ces résultats permettent d'attribuer les bandes de luminescence D-A correspondantes : l'étude de la variation des signaux de RMDO en fonction de la longueur d'onde a fourni un bon exemple du pouvoir de résolution de cette technique, permettant de séparer la luminescence self-activée de la luminescence des impuretés de cuivre.

5. ZnO : luminescence jaune. - De nombreuses études avaient été faites sur la luminescence jaune observée dans beaucoup d'échantillons de $\mathrm{ZnO}$ [28] avant qu'on ne se soit rendu compte qu'elle était liée à la présence d'une impureté acceptrice, le lithium $[29,30]$. Ceci a suggéré des études de $\operatorname{RMDO}[31,32]$ qui ont démontré que cette luminescence est une luminescence $\mathrm{D}$-A correspondant à une recombinaison donneur peu profond-accepteur lithium. Le spectre de RMDO ressemble encore à celui de la figure 3 puisque le lithium est un accepteur profond dans $\mathrm{ZnO}\left(E_{\mathrm{A}}\right.$ serait de l'ordre de $\left.0,7 \mathrm{eV}[29,30]\right)$. Ce système montre de fortes variations de la forme de la raie de RMDO en fonction de la puissance hyperfréquence, ce qui constitue pour la théorie de la largeur de raie un élément d'information très important.

6. ZnSe dopé au phosphore. - Le phosphore sur site sélénium $\mathrm{P}_{\mathrm{Se}}$ se comporte en accepteur profond dans $\mathrm{ZnSe}$ : les études de RPE classique sous photo- excitation effectuées par Watts et coll. [33] avaient montré que cet accepteur subit une distorsion JahnTeller trigonale. Une bande large de luminescence centrée vers $1,9 \mathrm{eV}$ avait été attribuée à une transition interne de l'accepteur $P_{S e}$ [34]. Les expériences de RMDO de Nicholls et Davies [35] ont démontré qu'il s'agit en fait ici encore d'une luminescence D-A, entre un donneur peu profond et l'accepteur phosphore. La conjonction des résultats de RPE classique, de luminescence et de RMDO permet d'affirmer que le niveau accepteur du phosphore est à $0,6 \mathrm{eV}$ environ de la bande de valence et non à $0,08 \mathrm{eV}$ environ comme le proposent Bhargava et coll. [36, 37].

7. ZnS dopé au gallium. - Ceci constitue le premier cas où un donneur profond a été étudié par cette technique (dans un semiconducteur). Pour le spectroscopiste de RPE, le signe marquant d'un donneur (cationique) profond est une interaction hyperfine importante avec le spin $I$ du noyau de l'impureté (dans le cas évidemment où $I$ est non-zéro). En effet, le spectre de RMDO obtenu dans $\mathrm{ZnS}$ dopé au gallium [38] est dominé par l'interaction hyperfine géante avec les noyaux ${ }^{69} \mathrm{Ga}$ et ${ }^{71} \mathrm{Ga}$ : ceci donne des spectres assez particuliers, la quantité $M_{\mathrm{D}}$ dans l'équation (2) n'étant plus un bon nombre quantique.

Le travail cité [38] a démontré l'existence d'une luminescence $\mathrm{D}-\mathrm{A}$, gallium-lacune de zinc, centrée à $1,94 \mathrm{eV}$ (la profondeur du niveau $\mathrm{Ga}_{\mathrm{Zn}}$ serait de $0,4 \mathrm{eV}$ environ). On aurait pu penser qu'une recombinaison entre un électron très localisé et un trou très localisé ne serait observée que si les deux centres étaient de très proches voisins. Cependant, la largeur de raie RMDO étant de $35 \mathrm{G}$ environ, ce qui donne une limite supérieure pour l'interaction dipolaire entre les deux spins, on peut affirmer que $r_{\mathrm{DA}}$ ne peut être inférieur à $10 \AA$. Signalons que des recombinaisons radiatives à longue distance entre électrons et trous très localisés commencent à être signalées dans les cristaux ioniques et font le sujet d'études de RMDO [39-41].

8. L'accepteur cuivre dans les composés II-VI. Le cuivre étant une impureté très courante dans les composés II-VI (voir la communication de J. C. Pfister à ce congrès [42]), de nombreux phénomènes de luminescence y sont associés, créant une situation extrêmement confuse. Jusqu'ici, la RMDO n'a pas vraiment modifié cette situation. On a observé la RMDO de donneurs peu profonds sur certaines bandes de luminescence qui sont attribuées à la présence du cuivre dans $\mathrm{ZnSe}$ et dans $\mathrm{ZnS}$ [15, 25, 43], démontrant au moins que ce sont des luminescences D-A, mais l'observation du spectre de RMDO de l'accepteur profond $\mathrm{Cu}_{\mathrm{Zn}}$ n'a pas encore été signalée. Il semble que dans $\mathrm{ZnS}$ et $\mathrm{ZnSe}$, l'ion $\mathrm{Cu}^{2+}$ isolé n'est pas observable par RPE classique non plus [44].

9. Silicium amorphe. - En conjonction avec les études de luminescence, les expériences de RMDO 
qui sont en cours dans plusieurs laboratoires devraient aider à comprendre les propriétés des états localisés qui peuvent exister dans ce matériau. Des signaux de RMDO ont été observés $[45,46]$ mais l'interprétation des spectres dans un amorphe est évidemment extrêmement difficile. Morigaki et coll. [45] attribuent leurs raies de RMDO à des centres donneurs et accepteurs et en déduisent que la luminescence observée correspond d'une part à des transitions D-A, d'autre part à la recombinaison d'un trou libre avec l'électron d'un donneur.

10. Propriétés de l'état excité d'un système D-A. Nous avons axé cet article sur l'intérêt de la RMDO comme outil d'identification de centres luminescents puisque c'est son apport principal au problème de la caractérisation des matériaux semiconducteurs. Cependant, la RMDO fournit également des renseignements sur les propriétés magnétiques des états excités, renseignements qui ne pouvaient pas être obtenus autrement. Citons rapidement quelques sujets d'intérêt.

Les raies de RPE détectées par l'intermédiaire de luminescences D-A sont très larges, en général 10100 fois plus larges que celles observées par RPE classique dans les mêmes échantillons. Plusieurs observations ont permis de déduire qu'il s'agit ici d'un élargissement inhomogène dû à l'interaction d'échange entre le donneur et l'accepteur (signalons que ce sont des interactions très petites, inférieures au micro-eV). Ces observations sont très diverses : augmentation de l'intensité des signaux de RMDO quand on applique une modulation de champ haute fréquence (par exemple à $100 \mathrm{kHz}$ ) [47], élargissement des raies RMDO induit par une source hyperfréquence puissante dans au moins un cas (paires D-A dans $\mathrm{ZnO}: \mathrm{Li}[31,32])$, variation de la forme de la raie de RMDO lors d'expériences en temps résolu [48]. Enfin, des expériences de double-résonance électronique-électronique (ELDOR) détectée optiquement sur la luminescence self-activée de $\mathrm{ZnS}$ [49] montrent d'une manière très directe qu'un point donné dans l'aile de la raie de RMDO du donneur et le même point dans l'aile de la raie de l'accepteur correspondent à une sous-classe de paires $\mathrm{D}$-A caractérisées par la même interaction d'échange.

L'effet d'une interaction d'échange du type

$$
J=J \mathbf{S}_{\mathrm{D}} \mathbf{S}_{\mathrm{A}}
$$

entre le donneur et l'accepteur a été expliqué par ailleurs $[31,32,50]$; nous avons donc simplement indiqué sur la partie droite de la figure 2 le déplacement des niveaux sous l'action du paramètre $J$. Ces déplacements conduisent à un dédoublement des transitions de RPE 1-2 et 4-3 (renversements de spin du donneur) et également des transitions $1-3$ et 4-2 (renversements de $S_{\mathrm{A}}$ ). Puisque $J$ est une fonction de $r_{\text {DA }}$, qui est une variable statistique dans le cristal, ceci conduit à un élargissement des raies de RMDO. Remarquons qu'en RMDO on est sensible aux paires D-A dont le temps de vie est relativement court, c'est-à-dire à celles où $r_{\mathrm{DA}}$ est petit et où $J$ est donc relativement grand. Ceci explique que la raie de RMDO soit plus large que la raie de RPE détectée classiquement : la forme de cette dernière raie donne une meilleure idée de la vraie distribution des interactions $\mathrm{D}$-A.

Des études de la réponse transitoire de la luminescence d'un système de paires D-A lorsqu'on applique une impulsion hyperfréquence ont été effectuées pour étudier le comportement des quatre sous-niveaux de la figure $2[16,51]$. Ces expériences montrent que la distribution de population entre les quatre niveaux de la figure 2 n'est pas de type Boltzmann - tout au moins pour les paires D-A observées par RMDO (c'est-à-dire celles dont la durée de vie est de l'ordre de la milliseconde). Au contraire, on trouve que $N_{4}$ et $N_{1}$ sont plus grands que $N_{3}$ et $N_{2}$. Ceci vient du fait qu'au cours d'une excitation laser continue il s'accumule une population relativement grande de paires dans les états nonradiatifs. Ce résultat n'est pas étonnant étant donné que les temps de relaxation spinréseau de donneurs et d'accepteurs profonds sont relativement longs à la température de l'hélium liquide, pouvant valoir dans le cas des accepteurs plus d'une seconde. (Il y a donc une grande différence par rapport aux systèmes de paires donneur-accepteur peu profond, où la présence d'un moment orbital $L=1$ couplé aux vibrations du réseau confère à l'accepteur un temps de relaxation très court : dans de tels systèmes le type de spectre RMDO obtenu indique que les sous-états de l'état excité sont thermalisés [6,7].)

Dans le cas des paires donneur-accepteur profond, puisque le transfert de population effectué par une onde hyperfréquence se fait toujours du niveau le plus peuplé vers le niveau le moins peuplé, les transferts de population sont ceux schématisés par les flèches $(1 \rightarrow 2,4 \rightarrow 3,1 \rightarrow 3$ et $4 \rightarrow 2$ ) sur la figure 2 , conduisant donc dans tous les cas à une augmentation de l'intensité de la luminescence, résultat observé dans la pratique (voir Fig. 3)

11. Conclusion. - Pour résumer, on peut dire que la détection optique de la résonance magnétique a un intérêt double dans le domaine de la physique des centres profonds dans les semiconducteurs. D'une part, à partir des propriétés des spectres de RPE, il est possible d'identifier des centres de luminescence (donneurs et accepteurs), donc d'expliquer l'origine de phénomènes de luminescence souvent très mal compris. A un stade plus appliqué, ceci fournit des renseignements utiles pour la caractérisation des matériaux semiconducteurs. D'autre part, le spectre de RPE détecté optiquement renseigne sur les propriétés physiques de centres et de systèmes de centres distribués dans un cristal. Il nous semble donc que cette technique est appelée à se développer encore dans les prochaines années, en particulier vers l'étude d'autres types de luminescence et d'autres classes de matériaux semiconducteurs. 


\section{Bibliographie}

[1] Magnea, N., Bensahel, D., Pautrat, J. L. et Pfister, J. C., Phys. Status Solidi 94b (1979) 627.

[2] Bensahel, D., Magnea, N. et DupuY, M., communication présentée à ce congrès, Revue Phys. Appl. (sous presse).

[3] Edel, P., Hennies, C., Merle d'Aubigne, Y., Romestain, R., Twarowski, Y., Phys. Rev. Lett. 28 (1972) 1268.

[4] Cibert, J., Edel, P., Merle d'Aubigne, Y. et Romestain, R., Semicond. Insulators 3 (1978) 137 et 163.

[5] Le. Si Dang, Merle d’Aubigne, Y. et Rasoloarison, Y., J. Physique 39 (1978) 760.

[6] Brunwin, R. F., Cavenett, B. C., Davies, J. J. et Nicholls, J. E., Solid State Commun. 18 (1976) 1283.

[7] Dunstan, D. J., Cavenett, B. C., Brunwin, R. F. et Nicholls, J. E., J. Phys. C 10 (1977) L 361.

[8] Hermann, C. et Lampel, G., Phys. Rev. Lett. 27 (1971) 373.

[9] Weisbuch, C. et Hermann, C., Phys. Rev. B 15 (1977) 816.

[10] Boccara, A. C. et Fournier, D., communication privée.

[11] Dean, P. J., Prog. Solid State Chem. 8 (1973) 1.

[12] Voir par excmple HaM. F. S., dans Electron Paramagnetic Resonance, rédacteur Geschwind, S. (Plenum Press, New York) 1972.

[13] Schirmer, O. F., Solid State Commun. 18 (1976) 1345.

[14] James, J. R., Nicholls, J. E., Cavenett, B. C., Davies, J. J. et Dunstan, D. J., Solid State Commun. 17 (1975) 969.

[15] James, J. R., CavenetT, B. C., Nicholls, J. E., Davies, J. J. et Dunstan, D. J., J. Lumin. $12 / 13$ (1976) 447.

[16] Nicholls, J. E., Davies, J. J., Cavenett, B. C., James, J. R. et Dunstan, D. J., J. Phys. C 12 (1979) 361.

[17] Prener, J. S. et Williams, F. E., J. Chem. Phys. 25 (1956) 361.

[18] Kasai, P. H. et Отомо, Y., J. Chem. Phys. 37 (1962) 1263.

[19] SCHNeIDER, J. dans II-VI Semiconducting Compounds, rédacteur Thomas, D. G. (Benjamin Press, New York) 1967, p. 40.

[20] Holton, W. C., DE Wit, M. et Estre, T. L. dans comptes rendus 1966 International Symposium on Luminescence Editions THIEMIG Munich, p. 454.

[21] Watkins, G. D., Solid State Commun. 12 (1973) 589.

[22] Shionoya, S., Koda, T., Era, K. et Fujiwara, H., J. Phys. Soc. Japan 19 (1964) 1157.

[23] Era, K., Shionoya, S. et Washizawa, Y., J. Phys. Chem. Solids 29 (1968) 1827.

[24] Müller, K. A. et Schneider, J., Phys. Lett. 4 (1963) 288.

[25] Dunstan, D. J., Nicholls, J. E., Cavenett, B. C., Davies, J. J. et ReDdy, K. V., Solid State Commun. 24 (1977) 677.
[26] Nicholls, J. E., Dunstan, D. J. et Davies, J. J., Semicond. Insulators 4 (1978) 119.

[27] Watkins, G. D., Phys. Rev. Lett. 33 (1974) 223.

[28] Kröger, F. A. et Vink, H. J., J. Chem. Phys. 22 (1954) 250.

[29] Schirmer, O. F. et Zwingel, D., Solid State Commun. 8 (1970) 1559.

[30] Zwingel, D., J. Lumin. 5 (1972) 385.

[31] Block, D., Cox, R. T., Herve, A., Picard, R., Santier, C. et Helbig, R., Semicond. Insulators 4 (1978) 131.

[32] Cox, R. T., Block, D., Herve, A., Picard, R., Santier, C. et Helbig, R., Solid State Commun. 25 (1978) 77.

[33] Watts, R. K., Holton, W. .C. et DE Wit, M., Phys. Rev. B 3 (1971) 404.

[34] Reinberg, A. R., Holton, W. C., De Wit, M. et Watts, R. K., Phys. Rev. B 3 (1971) 410.

[35] Nicholls, J. E. et Davies, J. J., J. Phys. C 12 (1979) 1917

[36] BHARGAVA, R. N., communication présentée à l'International Congress Recombination Semicond., Southampton (1978).

[37] Bhargava, R. N., Herko, S. P. et Fitzpatrick, B. J., Bull. Amer. Phys. Soc. (May 1979) 402.

[38] Davies, J. J. et Nicholls, J. E., J. Phys. $C$ (sous presse).

[39] DelbecQ, C. J., Toyozawa, Y. et Yuster, P. H., Phys. Rev. B 9 (1974) 4497.

[40] Delbece, C. J., Dexter, D. L. et Yuster, P. H., Phys. Rev. B 17 (1978) 4765.

[41] Von Der Weid, J. P. et Aegerter, M. A., Solid State Commun. 27 (1978) 519.

[42] Pfister, J. C., Revue Phys. Appl. (sous presse).

[43] BLOCK. D.. à publier.

[44] Clerjaud, B. et Gelineau, A., Phys. Rev. B 16 (1977) 82.

[45] Morigaki, K., Dunstan, D. J., Cavenett, B. C., Dawson, P. et Nicholls, J. E., Solid State Commun. 26 (1978) 981.

[46] Lampel, G., Rosso, M., Solomon, I. et Dunstan, D. J., résultats non publiés.

[47] Davies, J. J., J. Phys. C 11 (1978) 1907

[48] Dawson, P. et Cavenett, B. C. et Sowersby, G., Solid State Electron. 21 (1978) 1451.

[49] BLoCK, D. et Cox, R. T., à publier.

[50] Davies, J. J., Nicholls, J. E. et Cavenetr, B. C., Semicond. Insulators 4 (1978) 101

[51] Dunstan, D. J. et Davies, J. J., J. Phys. C (sous presse). 